\title{
Point prevalence of non-melanoma and melanoma skin cancers in Australian surfers and swimmers in SE Queensland and Northern New South Wales
}

\author{
A/Professor Mike Climstein ${ }^{1}$ \\ ${ }^{1}$ Affiliation not available
}

October 19, 2021

Climstein, $\mathrm{M}^{1,2,3}$., Doyle, $\mathrm{B}^{1,4}$., Stapelberg, $\mathrm{M}^{1,4}$., Rosic, $\mathrm{N}^{5}$., Hertess, $\mathrm{I}^{4}$., Furness, $\mathrm{J}^{3}$., Simas, $\mathrm{V}^{3}$., Walsh, $\mathrm{J}^{6}$.

${ }^{1}$ Aquatic Based Research, Faculty of Health, Southern Cross University, Bilinga, Qld, Australia

${ }^{2}$ Physical Activity, Lifestyle, Ageing and Wellbeing Faculty Research Group, University of Sydney, Sydney, NSW, Australia

${ }^{3}$ Water Based Research Unit, Bond University, Robina, Qld, Australia

${ }^{4}$ Advanced Skin Care, John Flynn Specialist Centre, Tugan, Qld, Australia

${ }^{5}$ Biomedical Sciences, Faculty of Health, Southern Cross University, Bilinga, Qld, Australia

${ }^{6}$ Sport Science Institute, Sydney, NSW, Australia

\section{Corresponding Author:}

Assoc. Prof Mike Climstein Aquatic Based Research, Faculty of Health Southern Cross University (Gold Coast) Phone: +61 755893330 email: michael.climstein@scu.edu.au

\section{Background/purpose}

Surfing and swimming are two popular outdoor aquatic activities in Australia with an estimated 2.7 million surfers and three million swimmers however, these activities are associated with intermittent exposure to ultraviolet radiation which is causal to skin cancer development. Our aim was to determine point prevalence of pre-skin cancer (actinic keratosis (PSC)), non-melanoma (NMSC) and melanoma skin cancers (MSC) in Australian surfers and swimmers.

\section{Methods}

This study involved Australian surfers from South-East Queensland (Australia) screened by a skin cancer doctor to determine the point prevalence of non-melanoma and melanoma skin cancers.

\section{Results}

Of 171 participants (surfers 116, swimmers 55) significantly more surfers were identified with a skin cancer of any type versus swimmers ( $50 \%$ vs $27.3 \%$; OR 2.67 ; P <.005) with most the common being PSC $(44.7 \%$ vs $11.3 \%, \mathrm{P}<0.05)$ followed by basal cell carcinoma (BCC) $(24.2 \%$ vs $7.6 \%, \mathrm{P}<0.05)$. There was a total of 7 MSC (5.2\% vs $1.8 \%$, respectively, P $>0.05$ ). Rates per 100,000 of NMSC and MSC (respectively) were BCC $(27,568$ vs 18,181$)$, squamous cell carcinoma (SCC) in situ (20,690 vs 40,00), SCC (9,482 vs 7,272) and $\operatorname{MSC}(5,172$ vs 1,818$)$. 


\section{Conclusion}

Surfers and swimmers had consistently higher rates of PSC, NMSC and MSC than the Australian general population. Point prevalence of MSC (groups combined) was 76-fold higher than the Australian general population. These findings highlight the clinical importance to regularly screen patients who surf or swim for early detection and treatment of skin cancer.

Keywords : basal cell carcinoma; actinic keratosis; squamous cell carcinoma; surfing; swimming; ultraviolet radiation

\section{Introduction}

Surfing and swimming are two popular recreational aquatic activities in Australia, with an estimated 2.7 million surfers ${ }^{1}$ and three million swimmers nationwide. ${ }^{2}$ These activities are associated with intermittent exposure to ultraviolet radiation (UVR), which is recognized as a causal mechanism in the development of non-melanoma (NMSC) and melanoma skin cancer (MSC). ${ }^{3}$ Intermittent exposure to UVR has been well documented to lead to development of actinic keratosis (AK) lesions, non-melanoma basal cell carcinoma (BCC), squamous cell carcinoma (SCC) and melanoma skin cancer (MSC). ${ }^{4}$ Australia is recognized as having the highest incidence (per 100,000) of NMSC and MSC in the world. ${ }^{5}$

Ultraviolet radiation in southeast Queensland is the second highest with a UVR range 4-12 and a mean of 7.9. A UVR index of three or greater is recognized as requiring sun protection strategies for the prevention of skin cancer. ${ }^{6}$

Skin Cancer Australia reported approximately one-third of Australians (30.8\%) had skin cancer, making it the most common type of cancer in Australia. ${ }^{7}$ Of skin cancers, NMSC, particularly BCC was reported to have the highest age-standardized rate $(1,541$ per 100,000) followed by SCC (1,035 per 100,000) and MSC (53.5 cases per 100,000) ${ }^{8}{ }^{8} 9$ Melanoma skin cancers were the costliest in Queensland with the highest proportion of total paid Medicare services related to MSC ( $30 \%)$ compared to the proportion of Australian's living in Queensland. ${ }^{10}$

The prevalence of skin cancer in swimmers is poorly reported in the literature, with only a single study conducted in the Netherlands ${ }^{11}$ and none to date conducted in Australia. The paucity of research in swimmers is also mirrored when investigating skin cancer in Australian surfers. Climstein et al., ${ }^{12}$ conducted an online survey of 1,348 Australian surfers and reported BCCs as the most prevalent $(6.8 \%, 9,124$ per 100,000) followed by MSC $(1.4 \%, 1,854$ per 100,000) and SCCs $(0.6 \%, 2,670$ per 100,000). The MSC rate was more than 34 -fold that of the Australian general population (53.5 per 100,000). ${ }^{9}$ It should be noted that this study used a retrospective design and relied on the participant's ability to self-report diagnosed skin cancers. Given the limited evidence and the lack of objective testing methods we therefore, sought to determine the point prevalence of NMSC and MSC in surfers and outdoor swimmers in southeast Queensland and Northern New South Wales through whole-body skin cancer examination. Confirmation of skin cancer was attained via histopathology.

\section{Methods}

Study design

This was a cross-sectional study design incorporating a survey followed by whole-body skin cancer screening. Participants were recruited from notices sent to local general practitioners (GPs), surfing and swimming clubs in addition to local media coverage.

Survey

The survey consisted of five sections which included participants' physiological demographics (Section one), activity-specific demographics (peak UVR exposure; Section 2) 13,14 and history of skin cancer (Section 3). Section four was completed by a clinician following the screening and included Fitzpatrick skin type ${ }^{15}$, type(s) and location(s) of skin cancer(s). Section five detailed histopathology results. 


\section{Screening strategy}

Whole body skin checks were conducted using a handheld dermatoscope, completed by an accredited skin cancer doctor focusing upon AK, NMSC and MSC using the chaos and clues algorithm ${ }^{16}$ and prediction without pigment, a decision algorithm for non-pigmented skin malignancy. ${ }^{17}$ These methods have been shown to significantly increase the diagnostic accuracy of detecting AK, NMSC and MSC when used by experienced clinicians. A digital dermatoscope with 10x magnification and LEDHQ illumination (Heine Delta 30, Heine, Optotechnic GhbH, Herrsching, Germany) was used to inspect any skin lesions as dermoscopy (epiluminescence microscopy) significantly increases the diagnostic accuracy of detecting PSC, NMSC and MSC when used by experienced clinicians. ${ }^{18,19}$

Statistical analysis

Normality of data was assessed via kurtosis, skewness, Q-Q plots and the Kolmogorov-Smirnov test (with Lilliefors significance correction). Heteroscedasticity was assessed with Levene's inferential test. All statistical analyses were completed using IBM's Statistical

Package for Social Sciences (SPSS, Ver. 27.0) and included demographics, independent

sample t-tests, Chi-square tests and ANOVA (Bonferroni post-hoc test) were used to determine significance between groups. Multivariate analysis was conducted to control for selected confounding variables. A bivariate (two-tailed) Pearson correlation coefficient was used to determine relationships between selected outcome variables. Alpha was set a priori at $\mathrm{P}<.05$ to determine significance between groups.

Point prevalence was determined by the number of surfers or swimmers with a AK, BCC, SCC, SCC in situ or MSC divided by those in the same group without. The standardized rate per 100,000 was calculated as, for example, the number of surfers or swimmer participants identified with a particular skin cancer multiplied by 100,000 then divided by the total number of surfers or swimmers, respectively. ${ }^{20}$ Odds ratios (OR), a ratio of two sets of odds, were determined with scale data and calculated as the odds of those in the exposed group (for example surfers with a BCC) divided by all surfers. The OR of surfers to swimmers was then determined by the odds in surfers divided by the odds in swimmers. Comparative OR for the Australia general population were identified within the literature. ${ }^{8,9,21}$

Ethics approval

This study was approved by the Southern Cross University Human Research Ethics committee (11 May, 2020/047).

\section{Results}

\section{Sample characteristics/demographics}

A total of 171 participants (males $n=94$, surfers $n=116$, ) competed the survey and underwent a total body skin check for AK, BCC, SCC in situ, SCC, and MSCs. Swimmers and surfers had similar mean age, height, mass, body mass index (BMI) and body surface area (BSA). When groups were combined, there was a significant correlation between the total number of skin cancers identified during the screening and both age $(\mathrm{r}=0.41, \mathrm{P}<.001)$ and BMI $(\mathrm{r}=0.24, \mathrm{P}<.05)$.

Swimmers were more experienced $(+13.1 \%$ years); however, surfers had significantly greater UVR exposure $(+34.9 \%, \mathrm{P}<.05)$ as estimated via activity reported for the previous 12 months (total hours/year). Most surfers (93.3\%) surfed year-round with 100 percent of participants surfing during the summer months. There were fewer swimmers $(65.7 \%)$ who swam year-round; however, all swimmers also swam during the summer months.

Both groups reported completing some activity during peak UVR (surfers $42.8 \%$ vs swimmer $42.7 \%$ ). However, both groups were similar in their estimated percentage of activity completed during peak UV (surfers and swimmers 5-100\%) (Table 1). 
Insert Table 1 approximately here

Prevention and screening strategies

A significantly higher percentage of surfers reported using either a chemical and/or physical prevention strategy as compared to swimmers $(100 \%$ versus $92.7 \%$, respectively; $\mathrm{P}<.005)$. With regard to scalp protection, a higher percentage of swimmers utilized a swim cap as opposed to surfers wearing a surf hat (32.7\% versus $20.7 \%$, respectively; $\mathrm{P}<.001)$. Significantly more surfers $(80.2 \%$ vs $49.1 \%$, respectively; P $<.001)$ utilized a rashie as opposed to swimmers; however, there were no differences between groups with regards to sunscreen, zinc, and lip balm use, or the reapplication of sunscreen (surfers $44.0 \%$ vs swimmers $47.9 \%)$.

The majority of each group (surfers $63.8 \%$ vs swimmers $72.2 \%$ ) placed emphasis upon whom conducted the skin examinations with most (surfers $43.9 \%$ vs swimmers $54.2 \%$ ) choosing to see a skin cancer doctor, followed by their GP (Table 2). The majority of participants (surfers $97.4 \%$ vs swimmers 100\%) were selfreferred for their skin cancer screening in the previous year, with less than half of the participants (surfers, $43.3 \%$; swimmers $40.7 \%$ ) having undergone a skin cancer check within the previous 12 months.

Screening results

Both groups identified as having a history of skin cancer (surfers $41.4 \%$ vs swimmers $36.4 \%$, respectively) and a family history of skin cancer (surfers $52.6 \%$ vs swimmers $43.6 \%$ ). The majority of surfers and swimmers that experienced blistering sunburns as a child were significantly $(\mathrm{P}<0.05)$ more likely found to have at least one skin cancer (BCC, SCC, SCC in situ, MSC; $74.1 \%$ vs $69.0 \%$ ) identified in this study than those who did not experience blistering sunburns as children. Surfers reported a significantly higher $(+85.5 \%, \mathrm{P}$ $<.05)$ number of lesions of concern as compared to swimmers prior to the screening.

There was no difference $(\mathrm{P}>.05$ ) in experience (years surfing or years swimming) between groups however, there was a significant relationship between surfing experience (yrs) and the number of skin cancers identified $(\mathrm{r}=0.312, \mathrm{P}<.001)$. There was no difference in surfing or swimming total exposure hours and the number of skin cancers $(\mathrm{r}=-0.126, ; \mathrm{r}=0.065$, respectively). However, when groups were combined, there were more participants identified with skin cancers when investigating total aquatic activity exposure (Figure 1 (A), quartile 1 vs quartile 4). Likewise, with groups combined, there were more skin cancers identified with increased activity exposure (quartile 1 vs quartile 4, Figure 1 (B)).

Insert Figure $1 \mathrm{~A}$ and B approximately here

A total of 74 AKs were identified during the screening (surfers 59, swimmers 15) and 110 skin cancers (BCC, SCC, SCC in situ, MSC) (Table 3). A significantly $(\mathrm{P}<.005)$ greater number of surfers were identified with a skin lesion (PSC, NMSC, MSC) during the screening than swimmers (132 vs 52, respectively; OR 1.85, $95 \%$ CI 1.0 to 3.5). Surfers also had a higher number $(\mathrm{P}>.05)$ of AK and rate (per 100,000) compared to swimmers (50,862 vs 27,273 respectively, OR 2.76). Surfers also had a higher number of BCCs compared to swimmers $(27,568$ vs 18,181 respectively, OR 1.71). Conversely, swimmers had a higher rate of SCC in situ $(20,6903$ vs 40,000, OR 0.70). Surfers had a significantly $(\mathrm{P}<.05)$ higher number of SCCs and a higher rate of SCCs compared to swimmers (9,482 vs 7,272, OR 1.34). With regard to malignant melanomas, surfers had a higher rate as compared to the swimmers (5,172 vs 1,818, OR 2.95).

When compared to the Australian general population, surfers and swimmers had higher ORs, which includes BCCs (OR 17.9 and 11.8, respectively), SCCs (OR 9.2 and 7.0, respectively) and MSC (OR 96.7 and 34.0, respectively) (Table 4). .

A MANCOVA demonstrated statistically significant differences in skin cancer totals (total number of skin cancers, total AKs, BCCs, SCC, SCC in situ, melanoma) based upon history of skin cancer (F = 3.98, Pillai's Trace $=1.40, \mathrm{P}<.001)$ with history of skin cancer as a fixed factor and covariates of Fitzpatrick skin type and age. Adjusting the mean number of skin cancers observed for covariates (age and Fitzpatrick skin type), totals were higher for those with a history of skin cancer (mean with skin cancer history 7.24 vs. 3.95 with 
no skin cancer history, $\mathrm{P}=.012)$. A similar trend was observed for AKs (6.03 vs. 3.69, NS), total BCC (0.66 vs. $0.06, \mathrm{P}=.001)$, total $\mathrm{SCC}$ in situ (0.50 vs. $0.101, \mathrm{P}=.042$ ), total melanoma (0.62 vs. 0.44 , NS) and total SCC (0.04 vs $-0.01, \mathrm{P}=.024$, with the negative mean value due to adjustment to the mean by covariates).

Most skin cancers in surfers were located on the face (28.0\%) followed by the arm and back (12.1\% each), whereas in swimmers, the majority of skin cancers were identified on the face $(17.3 \%)$, followed by the arm and lower leg ( $15.4 \%$ each). The highest number of melanomas were identified in surfers $(n=6)$ and mainly located on the face $(n=2)$ and back $(n=2)$. There was a single melanoma identified on the back in a swimmer. When the groups combined, the majority $(42.9 \%)$ of melanomas were identified on the back in participants, followed by the face $(28.6 \%)$ (Table 3 ).

A total of 110 samples were sent for histopathology, and all (100\%) were confirmed positive as either NMSC (BCC, SCC, SCC in situ) or MSC.

Fitzpatrick skin type and skin cancer

With regard to Fitzpatrick skin type and number of skin cancers $(\mathrm{P}=0.013)$, the majority of skin cancers (groups combined) were seen in the Fitzpatrick skin type 4 (59.1\%), this was followed by Type 3 (19.9\%) and Type $5(15.8 \%)$ (Table 5). We did not have any participants with a Fitzpatrick Type 1 or 6 skin types. Surfers and swimmers presented with predominantly skin type 4 (57.8\% vs $61.8 \%$, respectively) and this was followed by skin type $3(21.6 \%$ and skin type $5(13.4 \%)$ in surfers and skin type $5(20.0 \%)$ and skin type 3 $(16.4 \%)$ in swimmers (Table 5).

Gender differences

There was no significant difference between genders with regard to hours per year surfing or swimming, however females spent a great amount of the time during peak UVR $(+47.6$ surfing, $+44.1 \%$ swimming). There was no difference regarding the use of any prevention strategies between genders (males $96.8 \%$, females $98.7 \%)$. Males reported more sunburns as a child (59.7\% vs $63.8 \%)$ and a higher percentage reported having a sunburn in the previous 12 months $(75.5 \%$ vs $62.3 \%)$. A significantly $(\mathrm{P}<.05)$ higher number of females reported using a tanning bed ( $85.1 \%$ vs $8.5 \%)$. A greater number of females reported a family history of skin cancer ( $55.8 \%$ vs $44.7 \%$ ). With regard to the number of skin cancers, there was no significant difference between males and females (BCC, SCC, SCC in situ, MSC).

\section{Discussion}

To the best of our knowledge, this research represents the largest skin cancer screening study conducted by a clinician in Australian surfers and swimmers to date. It is also the largest whole-body screening of surfers for skin cancer. Surfers had higher standardized rates (per 100,000) of AK, BCC, SCC and MSC as compared to swimmers, whilst swimmers had a higher standardized rate of SCC in situ. Both aquatic groups (individually and combined) had rates higher than the Australian general population for NMSC and MSC. 8,9

The point prevalence rate of PSC (surfers $37.1 \%$, swimmers $21.8 \%$ ) is within the estimated percent of the Australian general population for surfers $(37-55 \%)^{21}$; however, swimmers were below this rate. It should be noted that these pre-cancerous AK lesions are well recognized as being precursors to the development of BCCs and $\mathrm{SCCs}^{21}$. Therefore, it is reasonable that participants identified with AKs will likely develop BCC and/or SCC sometime in the foreseeable future. Dodds et $\mathrm{al}^{22}$ reported the progress of AK to SCC was approximately one to 10 percent over 10 years.

The standardised rates (per 100,000) of BCC, SCC and MSC were consistently higher than the standardized rates (BCC surfers 27,568, swimmers 18,181 versus 11.8; SCC surfers 9,482, swimmers 7,272 versus 1,035; melanoma surfers 5,172 , swimmers 1,818 versus 53.5) previously reported in the Australian general population. The rates reported are similar than rates (per 100,000) previously reported in Australian surfers surveyed by Climstein et al. (BCC 9,124; SCC 2,670; melanoma 1,854). ${ }^{12}$ 
Iannacone and colleagues ${ }^{23}$ previously investigated MSC in Australian adolescents and young adults based upon de-identified data from the Queensland Cancer Registry. They reported an annual incidence of 10.1 per 100,000 for invasive melanoma. Our equivalent MSC rate was 180 to 512-fold comparatively. Unfortunately, the study by Iannacone and colleagues ${ }^{23}$ focused upon tumour type and did not contain exposure metrics related to UVR. Climstein and colleagues ${ }^{12}$ investigated Australian surfers via a survey as opposed to clinical screenings. They reported a rate of 1,854 per 100,000, which is near identical to the rate found in this study in swimmers $(1,818)$; however, it is below the rate we identified in surfers $(5,172)$. Although Climstein et al., ${ }^{12}$ did not report experience, the surfers in this current study were approximately $20 \%$ older, with the exposure (hours surfing per year) similar to that reported in the current study (mean $305 \mathrm{vs} 330 \mathrm{hrs} / \mathrm{yr}$ ). The actual skin cancer rate is most likely under reported in the survey study ${ }^{12}$ as it relied upon participants self-reporting as opposed to the objective methods used in the current study. It is feasible the higher rate of MSC found in the surfers in the present study is attributed to a greater exposure over the lifetime and the methodology of clinical screening as opposed to a survey.

Moehrl and colleagues ${ }^{24}$ previously reported that beach and water sports (and sunburn) are independent risk factors for the development of BCC; it is reasonable that surfing and swimming were therefore, an independent risk factor for the increased rates seen in NMSCs and MSCs. Both groups reported a similar history of sunburns (surfers $62.1 \%$, swimmers $61.8 \%)$; however, swimmers reported a significantly $(\mathrm{P}=.03)$ higher number of sunburns $(n=446)$ as compared to surfers $(n=353)$. Regrettably, we did not inquire into the severity nor duration of the sunburns in participants. Additionally, AK, BCC and SCC have been shown to be associated with an increased risk (4.3-fold) of developing a $\mathrm{MSC}^{25}$, thereby increasing risk of MSC in our participants who when screened were clear of MSC.

Solar UVR exposure and resultant skin cancers are dependent upon a number of geographical, behavioural and genetic susceptibility factors. Ultra-violet radiation has been estimated to cause approximately $95 \%$ of MSCs in areas of high exposure. ${ }^{26}$ Despite the high usage of chemical and/or physical protection strategies, our participants had a high point prevalence of NMSC and MSCs, suggestive of the long-term detrimental effects of UVR exposure whilst surfing or swimming. Furthermore, there is a direct correlation between sun exposure and UVR exposure, which accounts for 95\% of skin cancer cases. This risk is maintained in those participants continuing to surf or swim, which is highly likely as no participants commented upon ceasing their aquatic activity based upon a positive skin cancer screening.

It is well recognized that Fitzpatric skin types 1 and 2 are at highest risk of developing skin cancer due to the reduced pigmentation, as pigmentation affords a natural protection from UVR exposure however, we found the greatest prevalence of skin cancer was in type 4 followed by type 3. Despite the widespread use of Fitzpatrick skin typing, it has been rarely applied in similar research. For example Climstein and colleagues ${ }^{12}$ assessed skin type however, simplified the Fitzpatrick skin type to only fair to black, contrary to its intended clinical application. Other related studies neglected to investigate Fitzpatrick skin type altogether ${ }^{27-30}$ therefore limiting comparisons to our findings.

Strengths and limitations

To the best of our knowledge this was the first study in Australia assessing the prevalence of skin cancer in surfers and swimmers via total body skin checks conducted by a clinician. Further strengths include that screening was conducted by a specialist as opposed to recall via a survey. Additionally, the confirmation of all (100\%) histopathology samples by commercial laboratory analysis.

Our participants had a high degree of homogeneity as they were from a limited geographic locale; however, this restrictive inclusion criteria limited the ability to extrapolate our findings to other surfers and swimmers within Australia or elsewhere. However, intermittent exposure to UVR is well documented in the literature as a casual mechanism for the development of NMSC and MSC and we believe our findings have wide, geographic relevance.

It has however, been reported that Fitzpatrick skin type is a confounding variable for the development of NSMC and $\mathrm{MSC}^{31}$. Although Fitzpatrick skin type was assessed by a clinician, we cannot determine the 
effect Fitzpatrick skin type had on the development of NMSC and MSC in our participants, rather recognize that skin type is recognized as contributory to the development of skin cancer. Additionally, we did not note ethnicity, which may account for the skew in our skin types of participants in our study.

A limitation of the present study was that the sample size was relatively small however, when compared to the very limited number of skin cancer screening studies that incorporated clinician screening, we did however, exceed the participant numbers in previously published similar studies. We also recognize that we did not account for confounding factors such as occupational and other UVR exposure (i.e., other outdoor activities) which would have contributed to the point prevalence's reported. Also, with regard to our Fitzpatrick skin typing, we did not inquire into ethnicities, which may account for the skew in skin types. This study was self-selected, as it was not possible to attain a complete list of surfers in the area, we were therefore not able to utilize random sampling and as such selection bias may have occurred. Additionally, as the total number of surfers and swimmers in the region is unknown, we cannot assess nor calculate a response rate and therefore determine the representativeness of our participants to their respective aquatic groups. We believe there is no confounding bias as we did not investigate casual relationships.

\section{Conclusions}

The literature regarding point prevalence of skin cancer in Australian swimmers is completely undocumented and poorly documented via skin cancer screening in Australian surfers. We believe this study adds insight and heightens the awareness of clinicians to screen their patients who participate in surfing and swimming for the early detection and treatment of pre-invasive or early-stage invasive MSCs for improved health outcomes and reduced mortality ${ }^{23}$. Patients should also be educated on the benefits of sun protection behaviour strategies such as chemical and physical barriers as they have been previously shown to be effective ${ }^{24,32}$.

\section{Acknowledgements}

We would like to thank our participants for their time and dedication to our study. We would

also like to extend our sincere thanks to Professor Pat O'Shea, friend and mentor for instilling a passion for research; you are sincerely missed but not forgotten. MC, DB, NR, JF, VS and JW contributed to the study design, MS, IH and BD contributed to data collection, MC, BD, NR, JF, VS and JW contributed to data analyses and all authors contributed to the development of the final manuscript.

\section{References}

1. Stark A. Surfing Australia Annual Report. https://www.surfingaustralia.com/documents/annual\%20report\%202013.pdf. Published 2013. Accessed 20th August, 2014.

2. SportAus Ausplay. Swimming: State of Play Report. https://www.ascta.com/sites/default/files/assets/documents/Stateof-play-report.pdf. Published 2019. Accessed 29 December, 2020.

3. Jinna S, Adams BB. Ultraviolet radiation and the athlete: risk, sun safety, and barriers to implementation of protective strategies. Sports Med.2013;43(7):531-537.

4. Kanavy HE, Gerstenblith MR. Ultraviolet radiation and melanoma. Semin Cutan Med Surg.2011;30:222228.

5. Elflein J. Rates of skin cancer in the countries with the highest rates of skin cancer worldwide in 2018 https://www.statista.com/statistics/1032114/countries-with-the-greatest-rates-of-skin-cancer/. Published 2020. Accessed 28 December, 2020.

6. Gies P, Roy C, Javorniczky J, Henderson S, Lemus-Deschamps L, Driscoll C. Global Solar UV Index: Australian measurements, forecasts and comparison with the UK.Photochem Photobiol. 2004;79(1):32-39.

7. Cancer Council of Australia. Skin Cancer Statistics and Issues: Skin cancer incidence and mortality. https://wiki.cancer.org.au/skincancerstats_mw/index.php?title=Skin_cancer_incidence_and_mortality\&oldid=. Published 2020. Accessed. 
8. Lai V, Cranwell W, Sinclair R. Epidemiology of skin cancer in the mature patient. Clin Dermatol.2018;36(2):167-176.

9. Australian Institute of Health and Welfare. Cancer Data in Australia; Australian Cancer Incidence and Mortality (ACIM) books: melanoma of the skin. https://www.aihw.gov.au/reports/cancer/cancer-data-inaustralia/contents/summary. Published 2020. Accessed 2 January, 2021.

10. Australian Institute of Health and Welfare. Skin cancer in Australia. https://www.aihw.gov.au/getmedia/0368fb8b-10ef-4631-aa14-cb6d55043e4b/18197.pdf.aspx?inline=true.

Published 2016. Accessed 2 January, 2021.

11. Nelemans PJ, Rampen FH, Groenendal H, Kiemeney LA, Ruiter DJ, Verbeek AL. Swimming and the risk of cutaneous melanoma. Melanoma Res. 1994;4(5):281-286.

12. Climstein M, Furness J, Hing W, Walsh J. Lifetime prevalence of non-melanoma and melanoma skin cancer in Australian recreational and competitive surfers. Photodermatol Photoimmunol Photomed. 2016;32(4):207-213.

13. NSW Government: SafeWork. UVR is present every day, throughout the daylight hours, in varying levels of intensity. https://www.safework.nsw.gov.au/resource-library/heat-and-environment/the-risks-ofexposure-to-extreme-heat-and-uvr-when-working-outdoors-important-facts-to-know/exposure-to-extremeheat-accordions/3.-uvr-is-present-every-day,-throughout-the-daylight-hours,-in-varying-levels-of-intensity. Published 2020. Accessed.

14. Gonzaga ER. Role of UV light in photodamage, skin aging, and skin cancer: importance of photoprotection.Am J Clin Dermatol. 2009;10 (Suppl 1):19-24.

15. Gupta V, Sharma VK. Skin typing: Fitzpatrick grading and others. Clin Dermatol.2019;37(5):430-436.

16. Rosendahl C, Cameron A, McColl I, Wilkinson D. Dermatoscopy in routine practice - 'chaos and clues'.Aust Fam Physician. 2012;41(7):482-487.

17. Rosendahl C, Cameron A, Tschandl P, Bulinska A, Zalaudek I, Kittler H. Prediction without Pigment: a decision algorithm for non-pigmented skin malignancy. Dermatol Pract Concept. 2014;4(1):59-66.

18. Dinnes J, Deeks JJ, Chuchu N, et al. Dermoscopy, with and without visual inspection, for diagnosing melanoma in adults. The Cochrane database of systematic reviews.2018;12(12):Cd011902.

19. Dinnes J, Deeks JJ, Chuchu N, et al. Visual inspection and dermoscopy, alone or in combination, for diagnosing keratinocyte skin cancers in adults. The Cochrane database of systematic reviews. 2018;12(12):Cd011901.

20. Statistics Canada. Age-standardized rates. https://www.statcan.gc.ca/eng/dai/btd/asr. Published 2017. Accessed 11 August, 2021.

21. Smith Y. Actinic Keratosis Epidemiology. https://www.news-medical.net/health/Actinic-KeratosisEpidemiology.aspx. Published 2019. Accessed 10 January, 2021.

22. Dodds A, Chia A, Shumack S. Actinic keratosis: rationale and management. Dermatol Ther (Heidelb). 2014;4(1):11-31.

23. Iannacone MR, Youlden DR, Baade PD, Aitken JF, Green AC. Melanoma incidence trends and survival in adolescents and young adults in Queensland, Australia. Int $J$ Cancer. 2015;136(3):603-609.

24. Moehrle M. Outdoor sports and skin cancer. Clin Dermatol. 2008;;26(1):12-15.

25. Wernli KJ, Henrikson NB, Morrison CC, Nguyen M, Pocobelli G, Whitlock EP. Screening for Skin Cancer in Adults: An Updated Systematic Evidence Review for the U.S. Preventive Services Task Force. Agency for Healthcare Research and Quality (US). Screening for Skin Cancer in 
Adults: An Updated Systematic Evidence Review for the U.S. Preventive Services Task Force Web site. https://www.ncbi.nlm.nih.gov/books/NBK379854/. Published 2016. Accessed 14 March, 2021.

26. Armstrong BK, Kricker A. How much melanoma is caused by sun exposure? Melanoma Res.1993;3(6):395-401.

27. Dozier S, Wagner RF, Jr., Black SA, Terracina J. Beachfront screening for skin cancer in Texas Gulf coast surfers. South Med J. 1997;90(1):55-58.

28. Lawler S, Spathonis K, Eakin E, Gallois C, Leslie E, Owen N. Sun exposure and sun protection behaviours among young adult sport competitors. Aust N Z J Public Health.2007;31(3):230-234.

29. Zalaudek I, Conforti C, Corneli P, et al. Sun-protection and sun-exposure habits among sailors: results of the 2018 world's largest sailing race Barcolana' skin cancer prevention campaign. J Eur Acad Dermatol Venereol.2020;34(2):412-418.

30. Noble-Jerks J, Weatherby RP, Meir R. Self-reported skin cancer protection strategies and location of skin cancer in retired cricketers: a case study from membership of the Emu Cricket Club. J Sci Med Sport. 2006;9(6):441-445.

31. Ting W, Schultz K, Cac NN, Peterson M, Walling HW. Tanning bed exposure increases the risk of malignant melanoma. Int $J$ Dermatol. 2007;46(12):1253-1257.

32. Sander M, Sander M, Burbidge T, Beecker J. The efficacy and safety of sunscreen use for the prevention of skin cancer. CMAJ. 2020;192(50):E1802-e1808.

Table 1. Participant's demographics, values are mean \pm SD, number or percent, 95\% CI)

\begin{tabular}{ll}
\hline Parameter & Group $(\mathbf{n}=\mathbf{1 7 1})$ \\
\hline Age $($ years $)$ & $43.9(14.8)$ \\
Mass $(\mathrm{kg})$ & $74.7(14.3)$ \\
$\mathrm{BMI}\left(\mathrm{kg} / \mathrm{m}^{2}\right) *$ underweight $(\mathrm{n}) *$ normal $(\mathrm{n}) *$ overweight $(\mathrm{n}) *$ obese $(\mathrm{n})$ unreported & $24.5(3.4) 110450151$ \\
$\mathrm{BSA}\left(\mathrm{m}^{2}\right)$ & $1.89(0.2)$ \\
Experience $($ years $) *$ hrs $/$ wk $*$ wks $/ \mathrm{yr} *$ total/yr & $25.8(17.0) 6.6(4.9) 42.1(11.9) 29$ \\
UVR $*$ activity during peak UV (yes, \%) $*$ Activity percentage during peak UV $(\%)$ & \\
Lifetime aquatic hours & \\
\hline
\end{tabular}

Table 2. Participant's demographics (vales are percent). Where ${ }^{*}=\mathrm{P}<.05$.

\section{Parameter}

Prevention and Screening demographics

Uses any prevention strategy (yes, \%)

Uses hat* surf hat or swim cap (\%)

Uses rashie or wetsuit * yes $(\%)$

Previously underwent skin check $* 6$ months $(\%) * 1$ year ago $(\%) * 2$ years ago $(\%) * 3$ years ago $(\%) * 4$ years ago $(\%)$ Who performed last skin check $(\%) * \mathrm{GP} *$ Skin cancer doctor $*$ Dermatologist * Plastic surgeon

Table 3. Participant's history related to skin cancer (vales are number (n) or percent. Where ${ }^{*}=\mathrm{P}<0.05$

\begin{tabular}{llll}
\hline Parameter & Group $(\mathbf{n}=171)$ & Surfers $(\mathbf{n = 1 1 6})$ & $\mathbf{S}$ \\
\hline Personal history of skin cancer (yes, \%) & 39.8 & 41.3 & 3 \\
Family history of skin cancer (yes, \%) & 49.7 & 52.6 & 4
\end{tabular}




\begin{tabular}{|c|c|c|}
\hline Parameter & Group $(n=171)$ & Surfers $(n=116)$ \\
\hline History of blistering sunburns as a child (yes, \%) & 62.0 & 62.1 \\
\hline Number of sunburns in previous 12 months (n) & 589 & 248 \\
\hline Any lesions of concern (yes, \%) & 31.6 & 37.1 \\
\hline Personal history of skin cancer and skin cancer during screening (yes, \%) & 29.2 & 31.9 \\
\hline Skin cancer identified during screening (yes, \%) & 42.7 & 50.0 \\
\hline Scalp * Actinic keratosis * $\mathrm{BCC} * \mathrm{SCC}$ in situ $* \mathrm{SCC} *$ Melanoma & $\mathrm{n}=1081100$ & $\mathrm{n}=751100$ \\
\hline Nose $*$ Actinic keratosis $*$ BCC $*$ SCC in situ $*$ SCC $*$ Melanoma & $\mathrm{n}=93141$ & $\mathrm{n}=631110$ \\
\hline Face ${ }^{*}$ Actinic keratosis $*$ BCC $*$ SCC in situ $*$ SCC $*$ Melanoma & $\mathrm{n}=46375022$ & $\mathrm{n}=37303022$ \\
\hline Lip $*$ Actinic keratosis $*$ BCC $*$ SCC in situ $*$ SCC $*$ Melanoma & $\mathrm{n}=2 \quad 002000$ & $\mathrm{n}=2000200$ \\
\hline Ear $*$ Actinic keratosis $*$ BCC $*$ SCC in situ $*$ SCC $*$ Melanoma & $\mathrm{n}=820.0420$ & $\mathrm{n}=6220220$ \\
\hline Neck $*$ Actinic keratosis $*$ BCC $*$ SCC in situ $*$ SCC $*$ Melanoma & $\mathrm{n}=1082000$ & $\mathrm{n}=87 \begin{array}{lllllll} & 1 & 0 & 0 & 0\end{array}$ \\
\hline Shoulder $*$ Actinic keratosis $*$ BCC $*$ SCC in situ $*$ SCC $*$ Melanoma & $\mathrm{n}=802600$ & $\mathrm{n}=5020300$ \\
\hline Chest $*$ Actinic keratosis $*$ BCC $*$ SCC in situ $*$ SCC $*$ Melanoma & $\mathrm{n}=62210.01$ & $\mathrm{n}=4120.00 .01$ \\
\hline Arm $*$ Actinic keratosis $*$ BCC $*$ SCC in situ $*$ SCC $*$ Melanoma & $\mathrm{n}=2448651$ & $\mathrm{n}=1637231$ \\
\hline Back $*$ Actinic keratosis $*$ BCC $*$ SCC in situ $*$ SCC $*$ Melanoma & $\mathrm{n}=2101016023$ & $\mathrm{n}=16013012$ \\
\hline Hand $*$ Actinic keratosis $*$ BCC $*$ SCC in situ $*$ SCC $*$ Melanoma & $\mathrm{n}=13 \quad 10003000$ & $\mathrm{n}=10800200$ \\
\hline Upper leg $*$ Actinic keratosis $*$ BCC $*$ SCC in situ $*$ SCC $*$ Melanoma & $\mathrm{n}=502300$ & $\mathrm{n}=3012200$ \\
\hline Lower leg * Actinic keratosis * $\mathrm{BCC} * \mathrm{SCC}$ in situ $*$ SCC $*$ Melanoma & $\mathrm{n}=17 \quad 0 \quad 2 \quad 13 \quad 2 \quad 0$ & $\mathrm{n}=900117 \begin{array}{llll}0 & 0\end{array}$ \\
\hline Foot $*$ Actinic keratosis $*$ BCC $*$ SCC in situ $*$ SCC $*$ Melanoma & $\mathrm{n}=501310$ & $\mathrm{n}=3000210$ \\
\hline Totals $*$ Actinic keratosis $*$ BCC $*$ SCC in situ $*$ SCC $*$ Melanoma & $\mathrm{n}=184744246157$ & $\mathrm{n}=132593224116$ \\
\hline
\end{tabular}

Table 4. Skin cancer type by group. Values are percent (and 95\% CI)..

\begin{tabular}{llll}
\hline Parameter & Surfers: Point prevalence & Swimmers: Point prevalence & Surfers: Standardized rate per 1 \\
\hline Actinic keratosis & $37.1 \%(25.9-48.1 \%)$ & $21.8 \%(9.5-34.1 \%)$ & 50,862 \\
BCC & $11.2 \%(5.1-17.2 \%)$ & $14.5 \%(4.5-24.6 \%)$ & 27,568 \\
SCC in situ & $13.8 \%(7.0-20.6 \%)$ & $30.9 \%(16.2-45.6 \%)$ & 20,690 \\
SCC & $1.7 \%(-0.6-4.1 \%)$ & $3.6 \%(0.1-3.5)$ & 9,482 \\
Melanoma & $5.2 \%(1.0-9.3 \%)$ & $1.8 \%(0.1-5.4 \%)$ & 5,172 \\
\hline
\end{tabular}

Table 5. Fitzpatrick skin type and number of skin cancers identified during screening.

\begin{tabular}{llllll}
\hline Number of skin cancers & Skin type 2 & Skin type $\mathbf{3}$ & Skin type $\mathbf{4}$ & Skin type $\mathbf{5}$ & Total \\
\hline 0 & 5 & 20 & 51 & 22 & $\mathbf{9 8}$ \\
1 & 0 & 8 & 24 & 1 & $\mathbf{3 3}$ \\
2 & 0 & 4 & 11 & 1 & $\mathbf{1 6}$ \\
3 & 0 & 0 & 7 & 2 & $\mathbf{9}$ \\
4 & 1 & 0 & 3 & 0 & $\mathbf{4}$ \\
5 & 2 & 0 & 3 & 0 & $\mathbf{5}$ \\
6 & 1 & 2 & 0 & 1 & $\mathbf{4}$ \\
7 & 0 & 0 & 0 & 0 & $\mathbf{0}$ \\
8 & 0 & 0 & 1 & 0 & $\mathbf{1}$ \\
9 & 0 & 0 & 1 & & \\
Total & & & & & \\
Surfers & & & & & \\
Swimmers & 91 & 34259 & 1016734 & 2716 & $\mathbf{1 7}$ \\
116 & & & & &
\end{tabular}




\section{\begin{tabular}{llllll}
\hline Number of skin cancers & Skin type 2 & Skin type 3 & Skin type 4 & Skin type 5 & Total
\end{tabular}}

55

Figure 1. Number of participants identified with a skin cancer per quartile (A) and number of skin cancer identified per quartile (B).
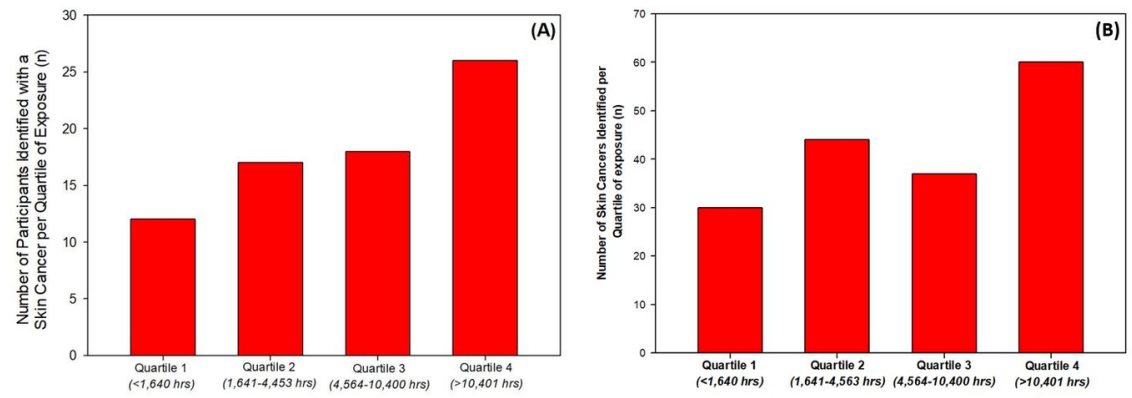